\title{
SIBRAGEC

\section{ANÁLISE COMPARATIVA DE APIS INTEGRADAS AO BIM PARA FOMENTO DA ECONOMIA CIRCULAR NA CONSTRUÇÃO'}

\author{
SANTANA, Ingrid Tainã Macário (1); MELO, Reymard Sávio Sampaio de (2); \\ ALBERTE, Elaine Pinto Varela (3); MIARA, Renata Degraf (4); SCHEER, Sergio
}

(1) Universidade Federal da Bahia, ingrid.taina@ufba.br (2) Universidade Federal da Bahia, reymard.savio@ufba.br (3) Universidade Federal da Bahia, elaine.varela@ufba.br (4)

Universidade Federal do Paraná, renatadmiara@gmail.com (5) Universidade Federal do Paraná, scheer@ufpr.br

\section{RESUMO}

A integração entre Interface de Programação de Aplicativos (API) e a Modelagem da Informação da Construção (BIM) possui grande potencial para facilitar a quantificação de resíduos na construção civil desde a fase de projeto. Deste modo, observa-se a possibilidade de uso de APIs para produzir informações de grande relevância para o fomento da economia circular (EC) na indústria da construção civil. Esse artigo comparou dois APIs e avaliou seus potenciais de uso para fomento dos princípios da EC no setor. O método adotado foi o mapeamento sistemático da literatura seguido de análise comparativa de dois APIs identificados na etapa anterior. A principal similaridade entre as APIs encontra-se no fato de ambas terem sido desenvoldidas para o software Autodesk Revit. A principal diferença está no uso das APIs em distintas etapas do ciclo de vida da edificação. Os resultados sugerem a necessidade de estudos que estendam a integração de APIs a diferentes softwares BIM.

Palavras-chave: Modelagem da Informação da Construção, Interface de Programação de Aplicativo, Economia Circular, Quantificação de resíduos.

\section{ABSTRACT}

The integration between Application Programming Interface (API) and Building Information Modeling (BIM) has great potential to facilitate quantifying construction waste from the design stage. Thus, there is the possibility of using APIs to produce highly relevant information to promote the circular economy $(C E)$ in the construction industry. This article compared two APIs and assessed their potential for use in furthering CE principles in the industry. The method adopted was the systematic mapping study followed by a comparative analysis of two APIS reported in the literature. The main similarity between the APIs lies in the fact that both were developed for Autodesk Revit software. The main difference is in the use of the APIs at different stages of the building lifecycle. The results suggest the need for studies that extend APIs integration to different BIM software.

Keywords: Building Information Modeling, Application Programming Interface, Circular Economy, Waste quantification.

\section{INTRODUÇÃO}

O crescimento das atividades de construção nos últimos anos tem produzido importantes quantidades de resíduos, acarretando diversos impactos ao meio ambiente. Os problemas mais notáveis que surgem da geração de resíduos na construção englobam a emissão de carbono e gases de efeito estufa, a imensa perda de energia e matérias-

\footnotetext{
${ }^{1}$ SANTANA, I. T, M.; MELO, R.S.S.de; ALBERTE, E.P. V.; MIARA, R.D.; SCHEER, S. Análise comparativa de APIs integradas ao BIM para fomento da economia circular na construção. In: SIMPÓSIO BRASILEIRO DE GESTÃO E ECONOMIA DA CONSTRUÇÃO, 12., 2021, Maceió. Anais[...] Porto Alegre: ANTAC, 2021. p. 1-8. Disponível em: https://eventos.antac.org.br/index.php/sibragec/article/view/516. Acesso em: 2 out. 2021.
} 
primas, o esgotamento dos aterros e o aumento dos custos do projeto (BILAL et al., 2015).

Adicionalmente, a desconsideração da gestão de resíduos de construção na etapa de planejamento de um projeto é um obstáculo importante para a efetiva adoção das práticas alinhadas aos princípios da Economia Circular (ESA; HALOG; RIGAMONTI, 2017; LU; YUAN, 2010; CHEN; LU, 2017).

A Economia Circular (EC) pode ser definida como um modelo econômico que promove maior eficiência no uso e gerenciamento de recursos da cadeia produtiva. A partir de ações de reutilização, recuperação, reparação e reciclagem, este modelo visa manter os materiais no seu ciclo produtivo (RIBEIRO; KRUGLIANSKAS, 2021; GHISELLINI; CIALANI; ULGIATI, 2016). Deste modo, a EC se destaca pela sua aptidão para minimizar os impactos ambientais da indústria da construção. Estudos, ações e tecnologias que visem a implementação de suas práticas devem ser incentivados.

Por outro lado, a adoção da Modelagem da Informação da Construção (BIM) durante a fase operacional do ciclo de vida dos ativos construídos possibilita reduzir substancialmente o custo e os materiais utilizados enquanto aumenta-se o desempenho e qualidade da edificação (DAVILA DELGADO; OYEDELE, 2020). A união dessas visões tem grande potencial para promover a redução da geração de resíduos nas fases de construção e desconstrução, causando menores sequelas econômicas, sociais e ambientais.

Diante disso, o objetivo desta pesquisa foi comparar duas Interface de Programação de Aplicativos (APIs) integradas ao BIM e avaliar os potenciais de uso dessas APIs para fomento dos princípios da EC no setor da construção civil.

\section{MÉTODO DE PESQUISA}

A pesquisa foi dividida em duas etapas: Identificação das APIs (Etapa 1) e Análise Comparativa das APIs identificadas (Etapa 2). Na Etapa 1 realizou-se um mapeamento sistemático da literatura (MSL) sobre o uso de BIM para EC, de caráter exploratório, com o objetivo de identificar APIs desenvolvidas para a gestão de resíduos na construção. O MSL tem como finalidade interpretar todas as pesquisas disponíveis e relevantes, a fim de aplicar uma metodologia de avaliação justa, confiável, rigorosa e aditável (KITCHENHAM, 2007).

Utilizando as bases Scopus e Web of Science, buscou-se artigos de periódicos internacionais sobre BIM e EC publicados entre 2009 e 2019 uma vez que o mapeamento teve início no ano de 2020. Para apoio a formulação da string de busca, adaptou-se o método PICO (Population, Intervention, Comparasion e Outcomes) proposto por Pai et al. (2004). Nessa pesquisa, somente os termos de busca inseridos em população (population) e intervenção (intervention) foram utilizados. A Figura 1 ilustra o protocolo de busca utilizado. A partir da leitura do título e dos resumos, chegou- se a uma amostra final de 125 artigos.

Em seguida, os artigos selecionados foram agrupados em sete categorias: Análise do ciclo de vida (30), Edifícios como Banco de Materiais (7), Consumo de energia (8), Desperdício de Materiais (13), Emissão de gases (21), Projeto para Desmontagem (24) e Barreiras a implementação ao uso do BIM (22).

A análise de conteúdo dos estudos primários desta categoria proporcionou seu detalhamento em três subcategorias, conforme ilustrado no Quadro 1. Para a Etapa 2 realizou-se uma análise comparativa entre o único estudo alocado na subcategoria "Utilização de API para quantificação dos resíduos" e o estudo de âmbito nacional de Miara e Scheer (2019) que propõe uma API integrada ao BIM para gestão de resíduos. Este estudo não foi identificado na busca, porém foi incluído a partir do conhecimento prévio dos autores. 
A análise comparativa buscou identificar as similaridades e diferenças das APIs. Para tal, considerou as seguintes variáveis de análise: objetivo principal da API, caráter de uso do modelo, forma de integração, etapas do ciclo de vida aplicáveis, contexto regional de uso, informações geradas, princípios aplicados a economia circular, oportunidades e funcionalidades. Finalmente, com as informações obtidas, analisou-se as potencialidades das APIs para fomento da EC na construção.

Figura 1 - Protocolo do mapeamento sistemático da literatura

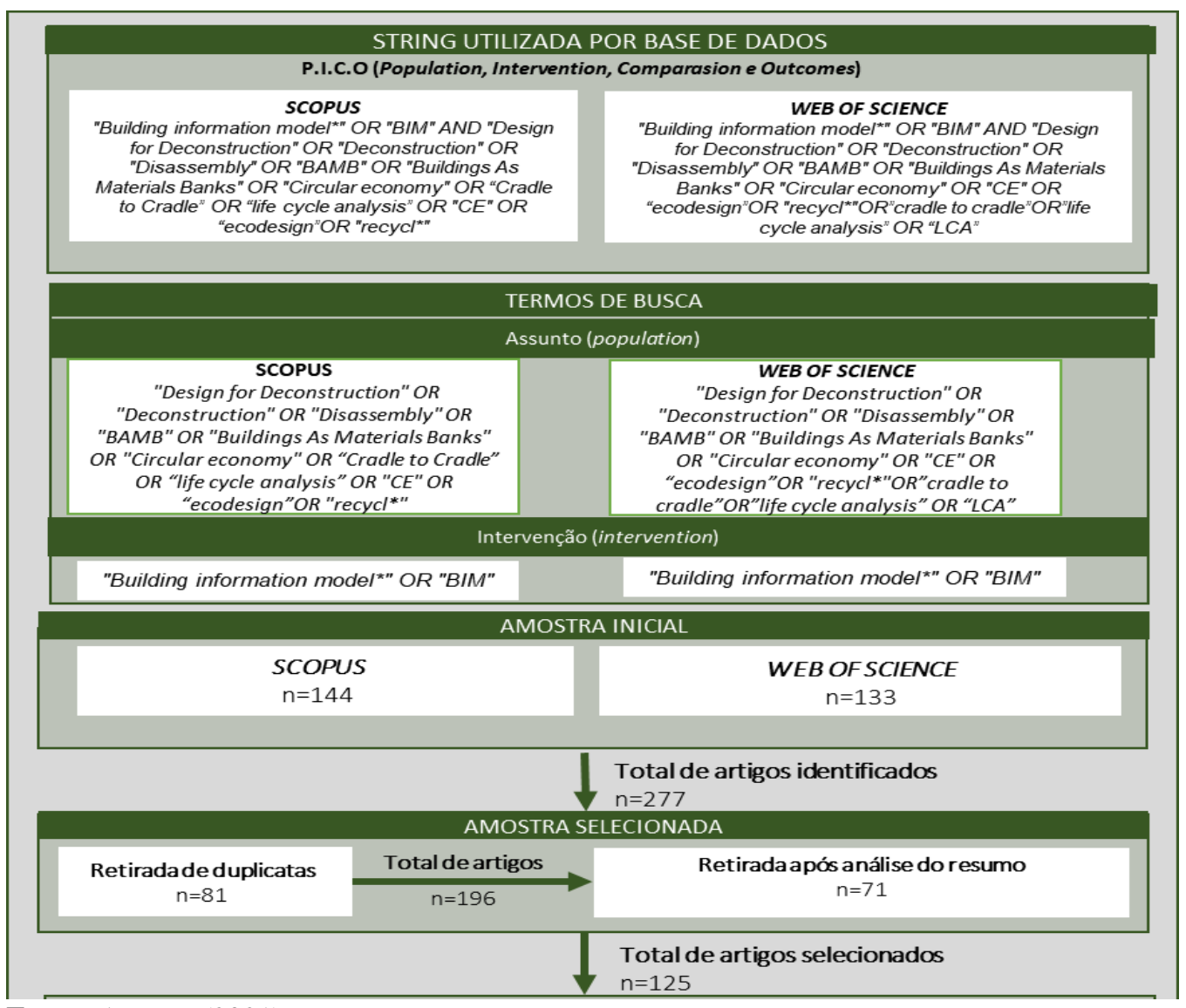

Fonte: Autores (2021)

Quadro 1 - Descrição das subcategorias

\begin{tabular}{|l|l|l|}
\hline \multicolumn{1}{|c|}{ Subcategoria } & \multicolumn{1}{|c|}{ Descrição } & \multicolumn{1}{|c|}{ Estudos Primários } \\
\hline $\begin{array}{l}\text { Gerenciamento } \\
\text { de resíduos de } \\
\text { construção }\end{array}$ & $\begin{array}{l}\text { Estudos primários acerca da necessidade de um } \\
\text { gerenciamento eficaz de resíduos de construção e } \\
\text { demolição em canteiros de obras, devido ao } \\
\text { crescimento da geração de resíduos e ao seu } \\
\text { redirecionamento para aterros sanitários, o que } \\
\text { contribui para a poluição na biosfera. }\end{array}$ & $\begin{array}{l}\text { Moreno (2019); } \\
\text { Gavali e Ralegaonkar (2018); } \\
\text { Bakchan et al. (2019); } \\
\text { Guerra et al. (2019); } \\
\text { Jalaei et al. (2019); } \\
\text { Jin et al. (2019); } \\
\text { Akinade e Oyedele (2019); } \\
\text { Ajayi et al. (2017); } \\
\text { Akbarnezhad e Nadoushani } \\
\text { (2015) }\end{array}$ \\
\hline $\begin{array}{l}\text { Ineficácias das } \\
\text { estratégias de } \\
\text { gestão de } \\
\text { resíduos }\end{array}$ & $\begin{array}{l}\text { Estudos primários acerca das dificuldades, entraves e } \\
\text { possíveis soluções para implantar estratégias de gestão } \\
\text { de resíduos, objetivando a redução detritos em aterros. }\end{array}$ & $\begin{array}{l}\text { Chidambaram (2019); } \\
\text { Alwan (2017); } \\
\text { Ajayi,et al. (2015) }\end{array}$ \\
$\begin{array}{l}\text { Utilização de } \\
\text { API para } \\
\text { quantificação } \\
\text { dos resíduos }\end{array}$ & $\begin{array}{l}\text { Estudo primário acerca do desenvolvimento de um } \\
\text { plug-in de API através do software BIM, visando a } \\
\text { automatização das informações sobre o ciclo de vida } \\
\text { da construção, de forma a quantificar os resíduos } \\
\text { esperados e os que foram gerados, visando minimizar } \\
\text { o impacto ambiental e financeira. }\end{array}$ & $\begin{array}{l}\text { Chileshe, Jayasinghe e } \\
\text { Rameezdeen (2019) }\end{array}$ \\
\hline
\end{tabular}

Fonte: Autores (2021) 


\section{RESULTADOS}

\subsection{Análise comparativa das APIs identificadas}

Ambos os estudos desenvolveram APIs integradas ao BIM, objetivando quantificar os resíduos gerados nos canteiros de obras, sendo possível comparar os efeitos dessa aplicação em dois cenários distintos.

Partindo desse pressuposto, entre as principais similaridades dos dois aplicativos estão o objetivo e os princípios da EC (Quadro 2). Ambos supriram a necessidade de uma API que tenha função de quantificar resíduos gerados na construção desde a fase de projeto, visando a reinserção deles no ciclo produtivo de forma mais automatizada. Além disso, observou-se que a adoção das duas APIs possui potencial para fomentar uma cadeia produtiva circular nos projetos, pois a partir das APIs propostas por Chileshe et al. (2019) e Miara e Scheer (2019) é possível:

- Planejar / identificar resíduos na etapa de construção e/ou demolição, proporcionando informações relevantes para a gestão adequada do resíduo produzido, de modo reinseri-lo na cadeia produtiva, a partir de reaproveitamento, reuso ou reciclagem;

- Identificar atividades críticas (que geram as maiores quantidades de resíduos no projeto), com vistas a desenvolver soluções sistemáticas para redução da geração do resíduo durante a etapa de construção;

- Realizar, durante a etapa de projeto, a análise comparativa entre distintas técnicas construtivas, para a identificação da melhor estratégia de construção no âmbito de geração de resíduos.

Quadro 2 - Similaridades entre as APIs

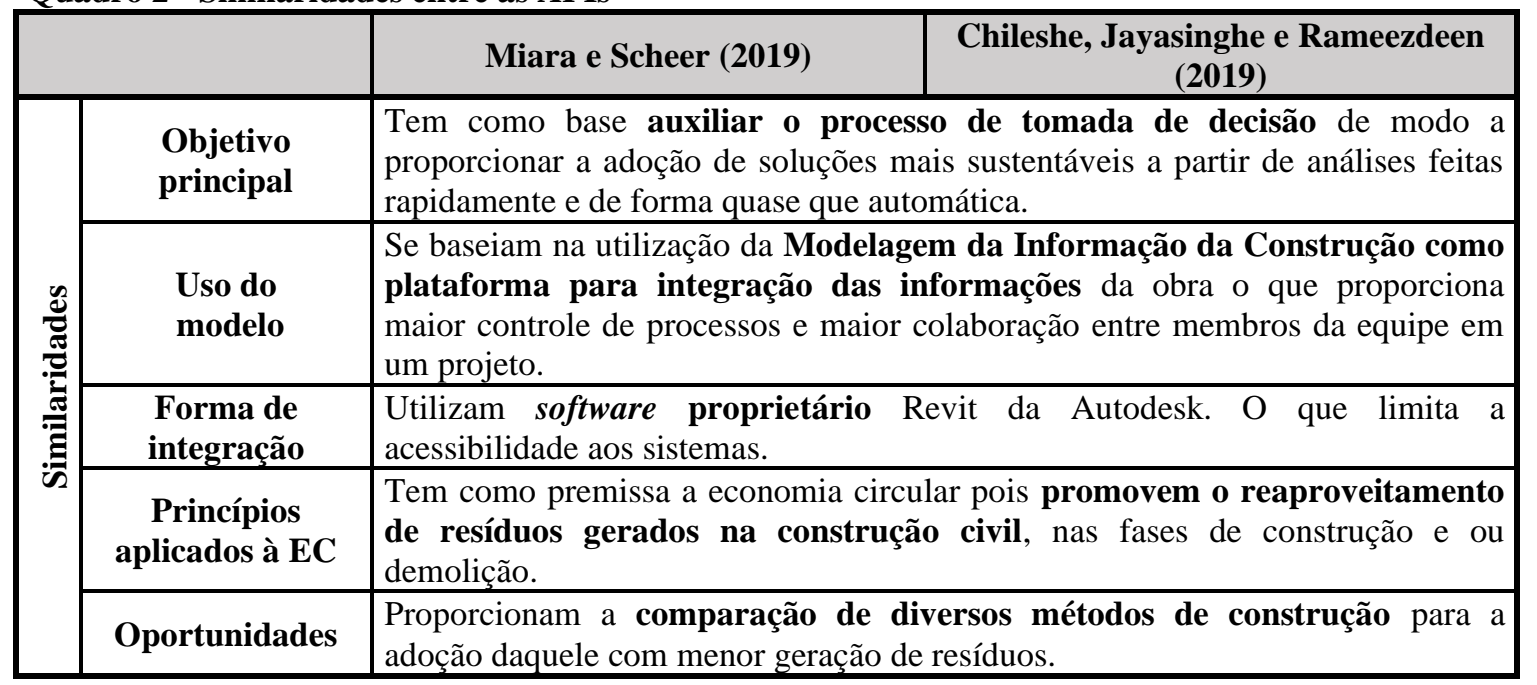

Fonte: Autores (2021)

Quanto as diferenças (Quadro 3), observa-se principalmente que as APIs em análise foram idealizadas para uso em distintas etapas do ciclo de vida da construção. A API de Miara e Scheer (2019) possui foco na consideração dos resíduos gerados na etapa de construção de edificações. Neste aplicativo somente são considerados resíduos de demolição de forma estimativa, para demolições que sejam necessárias no terreno onde se irá fazer uma nova construção. Seu principal objetivo é reduzir o desperdício de materiais na etapa de construção através de uma melhor gestão deles. O aplicativo também proporciona a análise e comparação de alternativas ou métodos construtivos considerando uma menor geração de resíduos.

Por sua vez, Chileshe, Jayasinghe e Rameezdeen (2019) tem foco na gestão dos resíduos gerados em processos de demolição e/ou desconstrução de edificações. Seu 
objetivo principal é de aproveitar os resíduos gerados nesse processo e separar aqueles que são perigosos para sua destinação correta, além de fornecer a possibilidade de testar a qualidade dos produtos reciclados.

Outro ponto abordado é a diferença entre os cenários, uma vez Chileshe, Jayasinghe e Rameezdeen (2019) abordam em seu artigo a indústria construtiva australiana, que possui maior nível de industrialização junto a processos off-site quando comparado a indústria brasileira. O baixo uso de processos off-site no contexto brasileiro faz com que as obras se tornem lentas e com maiores perdas de materiais, uma vez que sofre com as interferências climáticas e variações na produtividade dos trabalhadores.

Quadro 3 - Diferenças entre as APIs

\begin{tabular}{|c|c|c|c|}
\hline & Miara e Scheer (2019) & $\begin{array}{c}\text { Chileshe, Jayasinghe e Rameezdeen } \\
(\mathbf{2 0 1 9 )}\end{array}$ \\
\hline \multirow{4}{*}{ 窇 } & $\begin{array}{l}\text { Etapa do } \\
\text { ciclo de vida } \\
\text { aplicáveis }\end{array}$ & $\begin{array}{l}\text { Foco na etapa de construção: análise dos } \\
\text { resíduos de materiais que podem ser } \\
\text { reaproveitados e promoção de sua correta } \\
\text { destinação através de planejamento. }\end{array}$ & $\begin{array}{l}\text { Foco na etapa de desconstrução: } \\
\text { proporciona apoio a tomada de } \\
\text { decisões de aproveitamento de resíduos } \\
\text { e destinação correta inclusive daqueles } \\
\text { considerados perigosos. }\end{array}$ \\
\hline & \multirow{2}{*}{$\begin{array}{l}\text { Informações } \\
\text { geradas }\end{array}$} & $\begin{array}{l}\text { Volume e número de caçambas de } \\
\text { resíduos gerados durante a etapa de } \\
\text { construção por classe e pelo cronograma da } \\
\text { obra. }\end{array}$ & $\begin{array}{l}\text { Volume de resíduos gerados durante o } \\
\text { processo de desconstrução. }\end{array}$ \\
\hline & & $\begin{array}{l}\text { Proporciona a emissão de relatórios que } \\
\text { poderão ser utilizados para documentação } \\
\text { junto à órgãos regulamentadores assim } \\
\text { como durante a obra para controle. }\end{array}$ & $\begin{array}{l}\text { Gera os volumes e pesos de resíduos } \\
\text { gerados como índices absolutos que } \\
\text { podem ser comparados entre métodos } \\
\text { construtivos. }\end{array}$ \\
\hline & $\begin{array}{l}\text { Contexto } \\
\text { regional }\end{array}$ & $\begin{array}{l}\text { Brasileira: indústria construção civil ainda } \\
\text { possui pouco índice de industrialização. }\end{array}$ & $\begin{array}{l}\text { Australiana: maior índice } \quad \text { de } \\
\text { industrialização com } \\
\text { site. }\end{array}$ \\
\hline
\end{tabular}

Fonte: Autores (2021)

Sobre as funcionalidades em comum (Quadro 4), ambas APIs promovem a automatização da extração de dados do modelo BIM e a geração de resíduos por material, fornecendo maior controle de perdas de cada material no ciclo na construção e desconstrução. Contudo, entre as funcionalidades distintas, tem-se na API de Miara e Scheer (2019) a geração de resíduos por etapa do cronograma. Logo, esta informação possibilita identificar a fase da cadeia construtiva em que ocorre maior desperdício e o seu cronograma, bem como identificar a etapa e classe com maior geração de resíduos. Sendo assim, é possível resolver toda a problemática com maior antecedência, otimizando o tempo e os recursos. Todavia, na API de Chileshe, Jayasinghe e Rameezdeen (2019) têm-se a quantidade de resíduos perigosos gerados, sendo algo extremamente importante na etapa de desconstrução e/ou demolição, uma vez que, a partir disso, torna-se possível a separação entre os resíduos que serão reinseridos no ciclo produtivo e os que serão descartados.

Quanto ao potencial de uso das APIs para fomento da EC na construção, observa-se que ambos se complementam, visto que cada API possui foco em uma fase distinta. No entanto, destaca-se que a API de Miara e Scheer (2019) apresenta maior quantidade de dados para gestão e maior versatilidade para uso do modelo ao longo do ciclo de vida da construção para apoiar distintas práticas de fomento à EC (Quadro 5).

Ambas APIs não possuem estrutura para gestão direta dos resíduos produzidos ao longo da etapa de uso e manutenção da construção. 
Quadro 4 - Funcionalidades das APIs

\begin{tabular}{|c|c|c|}
\hline Funcionalidades & $\begin{array}{c}\text { Miara e } \\
\text { Scheer } \\
(2019)\end{array}$ & $\begin{array}{c}\text { Chileshe, } \\
\text { Jayasinghe e } \\
\text { Rameezdeen } \\
(\mathbf{2 0 1 9})\end{array}$ \\
\hline Dados extraídos de maneira automática do modelo BIM & $\mathbf{X}$ & $\mathbf{X}$ \\
\hline Resultados da geração de resíduos na etapa de construção & $\mathbf{X}$ & \\
\hline $\begin{array}{l}\text { Resultados da geração de resíduos na etapa de demolição ou } \\
\text { desconstrução }\end{array}$ & & $\mathbf{X}$ \\
\hline Resultados da geração de resíduos por material & $\mathbf{X}$ & $\mathbf{X}$ \\
\hline Resultados da geração de resíduos por classe de resíduo & $\mathbf{X}$ & \\
\hline $\begin{array}{l}\text { Resultados da geração de resíduos indicando quantidade de resíduos } \\
\text { perigosos gerados }\end{array}$ & & $\mathbf{X}$ \\
\hline Resultados da geração de resíduos por etapa do cronograma & $\mathbf{X}$ & \\
\hline $\begin{array}{l}\text { Possibilidade de alteração dos dados de índice de geração de resíduos } \\
\text { conforme dados obtidos experimentalmente ou em obra }\end{array}$ & $\mathbf{X}$ & NI \\
\hline $\begin{array}{l}\text { Emissão de relatórios que possibilitam a melhoria do gerenciamento da } \\
\text { obra e destinação correta dos resíduos gerados }\end{array}$ & $\mathbf{X}$ & \\
\hline
\end{tabular}

NI = Não identificado

Fonte: Autores (2021)

Quadro 5 - Potencialidades de uso das APIs para fomento da EC

\begin{tabular}{|c|c|c|}
\hline $\begin{array}{c}\text { Etapas / Práticas } \\
\text { da EC }\end{array}$ & Miara e Scheer (2019) & $\begin{array}{l}\text { Chileshe, Jayasinghe e } \\
\text { Rameezdeen (2019) }\end{array}$ \\
\hline $\begin{array}{l}\text { Planejamento e } \\
\text { projeto }\end{array}$ & $\begin{array}{l}\text { - Identificar melhor estratégia de } \\
\text { construção a partir de: } \\
\circ \quad \begin{array}{l}\text { Resultados da geração de } \\
\text { resíduos por material }\end{array} \\
\circ \quad \begin{array}{l}\text { Resultados da geração de } \\
\text { resíduos por classe de resíduo }\end{array} \\
\end{array}$ & $\begin{array}{l}\text { - Identificar melhor estratégia de } \\
\text { construção a partir de resultados } \\
\text { da geração de resíduos por } \\
\text { material }\end{array}$ \\
\hline Construção & 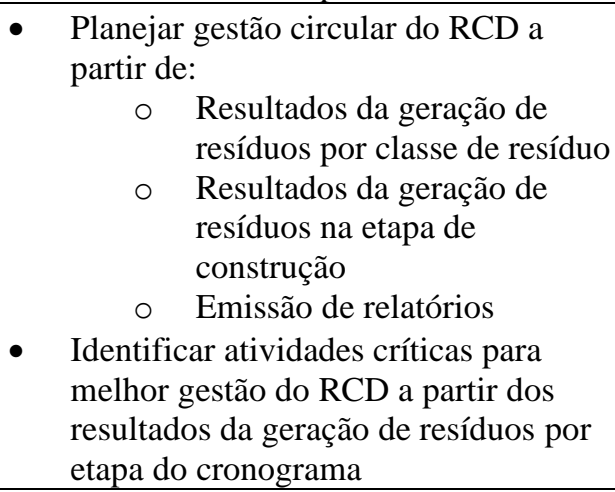 & NI \\
\hline Uso e manutenção & $\mathrm{NI}$ & $\mathrm{NI}$ \\
\hline Demolição & $\begin{array}{l}\text { - Planejar gestão circular do RCD a } \\
\text { partir de resultados da geração de } \\
\text { resíduos na etapa de demolição ou } \\
\text { desconstrução (uso de percentual de } \\
100 \% \text { de geração de RCD) }\end{array}$ & $\begin{array}{l}\text { - Planejar gestão circular do RCD } \\
\text { a partir de resultados da geração } \\
\text { de resíduos na etapa de } \\
\text { demolição ou desconstrução }\end{array}$ \\
\hline
\end{tabular}

NI = Não identificado

Fonte: Autores (2021)

\section{CONSIDERAÇÕES FINAIS}

Foi possível realizar uma comparação entre dois APIs integradas ao BIM visando quantificar os resíduos gerados na construção civil. Uma importante contribuição desse trabalho foi evidenciar a necessidade de automatização da construção civil através de APIs que gerenciem os índices de perda de materiais e analisem a sua reinserção na cadeia produtiva.

Dentre as principais similaridades entre as APIs, destaca-se o fato de ambos serem integrados ao software Revit. Entre as diferenças, se nota a finalidade principal para a 
qual a API foi desenvolvida. A API de Miara e Scheer (2019) possui o enfoque na geração de resíduos a partir da construção, ao contrário da API de Chileshe, Jayasinghe e Rameezdeen (2019), que foca nos resíduos gerados a partir da desconstrução.

Quanto as funcionalidades, ambas APIs se complementam, e possuem potencial para expandir seu uso dentro de um viés de fomento de uma cadeia produtiva circular na construção. Observa-se maior versatilidade de uso na API de Miara e Scheer (2019), pois, ainda que não tenha sido concebida diretamente para tal fim, a API proporciona informações que podem ser utilizadas para distintas práticas de fomento à EC.

Como em qualquer estudo, existem limitações para este artigo. Em primeiro lugar, o estudo foi limitado a artigos que tinham as palavras-chave da string de busca. Certamente, existem artigos que discutem os tópicos investigados, mas que não possuem essas palavras-chave. Em segundo lugar, a restrição de estudos da categoria "Desperdício de materiais" pode não contemplar algum estudo que utilize API com um foco em outro princípio da EC (eg. quantificação da emissão de carbono). Em terceiro lugar, o estudo se limita a análise de APIs ao software proprietário Revit da Autodesk. Pesquisas futuras poderiam estender a integração de API a outras tecnologias e a outros princípios da EC.

\section{REFERÊNCIAS}

AJAYI, S.O. et al. Attributes of design for construction waste minimization: A case study of waste-to-energy project. Renew. and Sust. Ener. Rev., v.73, 2017.

AJAYI, S.O. et al. Waste effectiveness of the construction industry: Understanding the impediments and requisites for improvements. Resources, Conservation and Recycling, v. 102, p. 101-112, 2015.

AKBARNEZHAD, A.; NADOUSHANI, Z.S.M. A computational method for selection of optimal concrete recycling strategy. Mag. of Conc. Reser., v. 67, p. 543-558, 2015.

AKINADE, O.O.; OYEDELE, L.O. Integrating construction supply chains within a circular economy: An ANFIS-based waste analytics system (A-WAS). Journal of Cleaner Production, v. 229, p. 863-873, 2019.

ALWAN, Z.; JONES, P.; HOLGATE, P. Strategic sustainable development in the UK construction industry, through the framework for strategic sustainable development, using Building Information Modelling. Journal of Cleaner Production, v. 140, 2017.

BAKCHAN, A.; FAUST, K.M.; LEITE, F. Seven-dimensional automated construction waste quantification and management framework: Integration with project and site planning. Resources, Conservation and Recycling, v. 146, p. 462-474, 2019.

BILAL, M.; OYEDELE, L.O.; QADIR, J.; MUNIR, K.; AKINADE, O.O.; AJAYI, S.O. Analysis of critical features and evaluation of BIM software: towards a plug-in for construction waste minimization using big data. International Journal of Sustainable Building Technology And Urban Development, v. 6, n. 4, p.211-228, 2015.

CHEN, X.; LU, W. Identifying factors influencing demolition waste generation in Hong Kong. Journal of Cleaner Production, v. 141, p. 799-811, 2017.

CHIDAMBARAM, S. Application of building information modelling for reinforcement waste minimisation. Proceedings of Institution of Civil Engineers: Waste and Resource Management, v. 172, p. 3-13, 2019. 
CHILESHE, N.; JAYASINGHE, R. S.; RAMEEZDEEN, R. Information flow-centric approach for reverse logistics supply chains. Aut. in Cons., v. 106, n. January, 2019.

DAVILA DELGADO, J. M.; OYEDELE, L. O. BIM data model requirements for asset monitoring and the circular economy. Journal of Engineering, Design and Technology, v. 18, n. 5, p. 1269-1285, 2020.

ESA, M. R.; HALOG, A.; RIGAMONTI, L. Strategies for minimizing construction and demolition wastes in Malaysia. Resour. Conserv. and Rec., v. 120, p. 219-229, 2017.

GAVALI, H.R.; RALEGAONKAR, R.V. Application of information modelling for sustainable urban-poor housing in índia. Proceedings of the Institution of Civil Engineers: Engineering Sustainability, v. 172, p. 68-75, 2018.

GUERRA, B.C.; BAKCHAN, A.; LEITE,F.; FAUST, K.M. BIM-based automated construction waste estimation algorithms: The case of concrete and drywall waste streams. Waste Management, v. 87, p. 825-832, 2019.

GHISELLINI, P.; CIALANI, C.; ULGIATI, S. A review on circular economy: the expected transition to a balanced interplay of environmental and economic systems. Journal of Cleaner Production. v. 114, p. 11-32, 2016.

JALAEI, F.; ZOGHI, M.; KHOSHAND, A. Life cycle environmental impact assessment to manage and optimize construction waste using Building Information Modeling (BIM). Inter. Jour. of Constr. Manag., v. 0, n. 0, p. 1-18, 2019.

JIN, R.; YUAN, H.; CHEN, Q. Science mapping approach to assisting the review of construction and demolition waste management research published between 2009 and 2018. Resources, Conservation and Recycling, v. 140, p. 175-188, 2019.

KITCHENHAM, B. A. Guidelines for performing systematic literature reviews in roftware engineering. Version 2.3, EBSE Technical Report, Keele University and University of Durham, UK, 2007.

LU, W.; YUAN, H. Exploring critical success factors for waste management in construction projects of China. Resour., Conserv. and Rec., v. 55, n. 2, 2010.

MIARA, R. D.; SCHEER, S. Optimization of construction waste management through an integrated BIM API. Iberoamerican Journal of Industrial Engineering, v. 11, p. $110-122,2019$.

MORENO, L.M.V. Sustainable constructions, environmental impacts. Nodo, v.14, p. 86-95, 2019.

PAI, M. et al. Systematic reviews and meta-analyses: an illustrated, step-by-step guide. Nat. Med. Jour. of Ind., v. 17, n. 2, p. 86-95, 2004.

RIBEIRO, F. M.; KRUGLIANSKAS, I. A Economia Circular no contexto europeu: conceito e potenciais de contribuição na modernização das políticas de resíduos sólidos. Disponível em: https://www.engema.org.br/XVIENGEMA/473.pdf Acesso em: 4 de fevereiro de 2021.

\section{AGRADECIMENTOS}

Os autores gostariam de agradecer ao CNPq pela bolsa de iniciação científica concedida a primeira autora. 